\title{
Sanderbildung durch subglaziäre, aufsteigende Schmelzwasserströme?
}

\author{
Von Paul J a s persen, Kiel
}

\begin{abstract}
Allgemein ging bisher die Anschauung über die Entwässerung des norddeutschen Inlandeises dahin, daß sie subglaziär wie supraglaziär in zentrifugaler Richtung erfolgt und daß nur so auch eine Erklärung der vor dem ehemaligen Eisrand liegenden Sander gegeben sei. Als Ursache des Aufsteigens des subglaziären Schmelzwassers aus der Mulde der Ostsee zur Höhe der Sander hinauf wird der hydrostatische Druck, unter dem das Schmelzwasser unterhalb des Eises stand, angenommen. Gegen diese Theorie erheben sich mehrfache Bedenken. Sie liegen zunächst auf morphologischem Gebiet; aber auch die physikalisch-hydrostatischen Fragen können noch keineswegs als geklärt gelten. Das Hinaufdrücken des Schmelzwassers auf die Höhe der Sanderflächen ist aber nicht nur ein hydrostatisches Problem, sondern gleichzeitig und mindestens ebenso sehr ein hydrodynamisches. Von dieser Seite her ist die obige Theorie noch kaum untersucht worden. Ein Versuch dazu soll im folgenden unternommen werden. Hierbei sind in erster Linie die Verhältnisseder C.imbrischen $\mathrm{Halbinsel}$ in Betracht gezogen worden.
\end{abstract}

\section{Fra.gen auf morphologis chem Gebiet}

Zunächst sollen einige Bedenken morphologischer Art kurze Erwähnung finden:

1.) Die Sander sind in fast ununterbrochener Erstreckung von Norden bis Süden vorhanden und zwar auch da, wo keine Ausmündungen von Tunneltälern vermutet werden können, weil die Oberflächenform keine Grundlage dazu bietet. Die Tunneltäler sind also nicht Voraussetzung der Sander.

2.) Es fehlt vielfach, namentlich in Nord- und Südschleswig, an einwandfreien Spuren einer Verbindung von den vermuteten Tunneltälern zu den nächstgelegenen, nach Westen führenden Wasserläufen, obgleich es sich bei den abzuleitenden Schmelzwassern um sehr große Mengen gehandelt haben müßte, deren Strombetten nicht ohne deutliche Spuren verschwinden würden. Die Tatsache allein, daß west- und ostwärts führende Wasserläufe vorhanden sind, deren Ursprungspunkte diesseits und jenseits der Wasserscheide in geringer, aber immerhin kilometerweiter Entfernung voneinanderliegen, stellt keinen Beweis dafür dar, daß es sich um zusammengehörende, früher durchgehende Wasserläufe handelt. Als Beispiel sei das vom Busdorfer Teich bei Schleswig südwärts streichende Tal genannt. Es endet in einem Talkessel, dessen umrahmende Höhen keine Lücken zeigen, die auf den früheren Durchlaß eines Wasserstroms hindeuten.

Wo aber ein früherer Auslauf nach Westen angenommen werden kann, wie mehrfach im holsteinischen Gebiet, besteht keine Nowendigkeit, ihn als Ausmündung eines Tunneltales zu erklären. Er ist als Überlauf eines beim Rückzug des Eisrandes gebildeten Stausees (.z B. Emkendorfer Senke beim Westensee bei Kiel) oder als direkter Abfluß supra- oder inglaziären Schmelzwassers zu deuten. 
3.) Fördenbecken mit Schwellen und innere Fördentäler mit Seen, die sich stufenweise aufwärts hintereinander reihen, werden in Anlehnung an die gleichen Erscheinungen in Skandinavien als Kennzeichen von Tunneltälern angesehen (z. B. E. Werth 1908/9, S. 346ff.). Der wesentliche Unterschied, daß im einen Falle Eis und Wasser abwärts, im anderen Falle nach der herrschenden Theorie aufwärts, also gegen die Stufen, liefen, schließt aber die Analogie zu den subglaziären Rinnentälern in Skandinavien aus.

4.) Viele der vermuteten Tunneltäler verdanken ihre Entstehung erst späteren, östlicher gelegenen Eisrandlagen, sie bestanden also noch garnicht zu der Zeit, als das Eis bis zu den Sandern reichte (z. B. innerstes Haderslebener Fördental und das Tal Krusau-Pattburg bei Flensburg). Ein von der hier behandelten Hypothese nicht beeinflußter Beobachter würde schwerlich ein Tal wie das Krusau-Pattburger als subglaziär durch aufwärtsfließende Schmelzwässer oder durch eine Eiszunge entstanden erklären, da das äußere Bild unzweifelhaft darauf hindeutet, daß die Talführung ein Ergebnis des später aufgeschütteten östlicheren Rückens darstellt. $\mathrm{Zu}$ beachten ist auch, daß dieses Tal rechtwinklig zur wahrscheinlichen Hauptbewegungsrichtung des Inlandeises von der Förde abzweigt, dann seine Richtung um etwa $150^{\circ}$ ändert und so zweimal von der Hauptbewegungsrichtung des Eises gekreuzt worden sein müßte. Es muß angenommen werden, daß das Eis den Tunnel zuschütten und einebnen würde.

5.) Die vermeintlichen Tunneltäler verengern sich nach oben zu Betten kleinster Rinnsale ohne Anzeichen, daß sie früher größere Wassermengen aufwärts geführt hätten; sie zeigen hier im Gegenteil alle Kennzeichen von Erosion durch abwärtsfließendes Wasser. Wenn sie Becken einer Gletscherzunge gewesen wären, dann vürde diese die aus weichem Boden bestehenden Stufen und Talverengungen beseitigt und ein mehr ausgeglichenes Tal hergestellt haben. (Die breiteren Täler mit Rinnenseen, weiter östlich gelegen, weisen auf Eiserosion hin, z. B. die Langsee-Oxbekrinne nördlich der Schlei.)

6.) Es ist damit zu rechnen, daß schon zur Zeit des Höchststandes der Weichseleiszeit das subglaziäre Schmelzwasser einen subglaziären Abfluß durch den Großen Belt oder den Sund zum Kattegatt und Skagerrak gehabt hat. Die morphologischen Verhältnisse der Ostseemulde und des Belts sprechen dafür, daß ein Gefälle nach Norden bestanden hat. Die Absenkung Skandinaviens während der Weichseleiszeit infolge des Eisgewichts hat sich auch auf die dänischen Inseln erstreckt, sodaß eine tiefere Lage des Beltgrundes zur damaligen Zeit anzunehmen ist; das subglaziäre Wasser konnte und mußte also der Schwerkraft folgend nach unten abfließen. Die Anschauung, daß sich an der Sohle des Inlandeises ein Tunnelsystem mit Schmelzwasser befunden habe, ist allgemein. Das Schmelzwasser wurde zum Teil durch den aus der Erde kommenden Wärmestrom erzeugt. Diese Menge war zwar sehr gering (Вrocкамp 1951, S. 54), aber man muß sie als grundlegende, allgemeine Ursache zur Bildung und zur dauernden Aufrechterhaltung einer Schmelzwasserschicht ansehen, die dann durch hinzutretende Oberflächen-Schmelzwässer vermehrt wurde. Man kann weder annehmen, daß sich dieses Entwässerungssystem auf einzelne Gebiete beschränkt habe, noch daß irgendwo Riegel bestanden, welche die durchgehende Verbindung unterbrochen hätten. Auch die Tiefenverhältnisse im Kattegatt und Skagerrak deuten darauf hin, daß ein subglaziärer Abfluß zum Atlantik bestanden hat. Wenn ein solcher aber bestand, konnte kein so hoher hydrostatischer Druck in der Ostseemulde erhalten bleiben, daß er zum Hinaufdrücken von Wasser und Gesteinsmaterial zu den Sandern genügt hätte. 
7.) Nachdrückliche Zweifel rufen die $100 \mathrm{~m}$ hochgelegenen Sander im nordwestlichen Jütland hervor. Sie liegen in nächster Nähe der Nordsee und damit in geringer Entfernung vom tiefen, nicht mehr vom geschlossenen Inlandeis bedeckten Meer. Daß das subglaziäre Schmelzwasser aus der großen Tiefe zu dem damals vielleicht $180 \mathrm{~m}$ über dem Meeresspiegel liegenden Sandergebiet empor steigen würde, statt westlich abwärts zum nahen Atlantik auszuweichen, erscheint unwahrscheinlich.

\section{Fragen auf hydrostatischem Gebiet}

Die Stärke des hydrostatischen Drucks in dem unterhalb des Eises befindlichen Schmelzwasser richtet sich nach der Höhe des Wasserspiegels in den subglaziären Wasserläufen bzw. in den bis zur Oberfläche reichenden Spalten. Maßgebend für den die Überwindung der Schwelle bewirkenden hydrostatischen Druck ist nur der Teil der Wasserhöhe in den Spalten, der über dem Niveau der Abflußöffnung auf der Schwelle liegt, und ferner kommt nur in Betracht die Wassersäule in den letzten Spalten, die sich in Richtung auf die Ausflußöffnung zu befinden, weil nach dem Gesetz der kommunizierenden Röhren der hydrostatische Druck aus höher gelegenen Spalten durch die tiefer gelegenen Entlastung erfährt.

Teilweise wird angenommen, daß das Inlandeis keine Spalten gehabt habe, die von der Ober- bis zur Unterfläche durchgingen. Ob diese Anschauung richtig ist, kann hier dahingestellt bleiben. Jedenfalls ist sie aber nicht vereinbar mit der Theorie, daß unter dem Eise ein hydrostatischer Druck bestanden habe, der zum Hinaufdrücken von Wasser und Gletscherschutt zu den Sandern genügt habe. Ohne Hinzunahme der Höhe der Eisdecke und entsprechende Erhöhung des Wasserspiegels in Spalten konnte kein genügend starker Druck erzeugt werden. Da zum anderen das subglaziär entstehende Schmelzwasser, wie alle Gletscher im Winter zeigen, nur verhältnismäßig geringe Mengen aufweist, muß der Zutritt supraglaziären Wassers auf dem Wege durchgehender Spalten vorausgesetzt werden, wenn eine genügende Schwemmwirkung (siehe unter III) erwartet werden soll. Ein solcher Zutritt ist naturgemäß wesentlich auf das Zehrgebiet des Inlandeises beschränkt.

\section{Fragen auf hydrodynamischem Gebiet}

In beiden oben berührten Fragen der Spaltenbildung und des Abflußes zum Kattegatt mögen die Meinungen geteilt sein. Um aber auf jeden Fall ein voreiliges Urteil $\mathrm{zu}$ vermeiden, soll in der folgenden hydrodynamischen Untersuchung zunächst noch in beiden Fragen die der bisherigen Hypothese günstigere Alternative zu Grunde gelegt werden. Es soll also zu m Z we cke de r Untersuchung zunächst vorausgesetzt werden, daß es Spalten mit Wasserführung von der Ober- bis zur Unterfläche gegeben habe und daß kein subglaziärer Abfluß zum Kattegatt bestanden habe. Es soll ferner die Wirkung randnäherer Spalten (siehe II Abs, 1) unbeachtet gelassen werden. P hy sikalisch gesehen würdeder größte, überhaupt denkbare hydrostatische Druck dann entstehen, wenn im Übergangsgebiet zwischen dem Nähr-und dem Zehrgebiet des Gletschers bis zum Rand mit Wasser gefüllte Spalten die Verbindung vom supraglaziären zum subglaziären Wasser hergestellt hätten.

Sichere Berechnungen der absoluten Höhe des hydrostatischen Drucks können schon deswegen nicht aufgestellt werden, weil nach obigen Darlegungen 
positive Angaben darüber, in welchem Höhenniveau die Entstehung des höchsten Drucks tatsächlich stattgefunden hätte, nicht gemacht werden können. Unter den obigen Voraussetzungen würde sich zwar ein hydrostatischer Druck von genügender Stärke ergeben, um das Wasser bis zu den Sandern hinaufzudrücken. Die Hypothese der Tunneltäler mit aufwärts gerichtetem Wasserstrom spricht aber nicht nur von einem Wasseraustritt, sondern auch von einer kräftigen Sand- und Schuttförderung, die zur Aufschüttung der Sander diente. Zu einer solchen Förderung ist aber eine beträchliche Geschwindigkeit des schiebenden Wasserstroms erforderlich. Zur Beantwortung der Frage nach der Was-sergeschwindigkeit müßte zunächst das Gefälle des Wasserlaufs bekannt sein. Das Gesamtgefälle des subglaziären Wasserstroms in dem oben geschilderten günstigsten Fall ist genau gleich dem Gesamtgefälle der darüberliegenden Eisoberfläche, da die Anfangs- und Endpunkte beider dieselben sind. Das Gefälle der Eisoberfläche kann mit recht großer Sicherheit veranschlagt werden: Aus einer höchsten Höhe des Eises von etwa $3000 \mathrm{~m}$ auf der schwedischen Eisscheide und einer Weglänge von etwa $1000 \mathrm{~km}$ ergibt sich ein Gesamtgefälle von 3\%. Wenn man die Herkunft des Eises aus dem Bottnischen Busen annimmt, so würde der Weg von dort über Südschweden noch länger und das Gefälle entsprechend kleiner sein. Diés wäre allerdings das Gefälle ,in der Luftlinie“. Da das Wasser aber unter dem Eise nicht auf dem kürzesten Wege nach Westen geflossen sein wird, sondern zweifellos in Windungen und Umwegen (siehe unten c), so wird das Gefälle des tatsächlichen Weges wesentlich geringer zu veranschlagen sein.

Es wird demgegenüber aber geltend gemacht, daß im Abschmelzgürtel ein sehr viel höheres Gefälle bestanden haben müsse, manchmal unter Berufung auf grönländische und isländische Verhältnisse. Ein Vergleich mit einem Inlandeis, das wie in den dortigen Gebieten über mehr oder weniger steilem Untergrund $a \mathrm{~b}$ wärts fließt, ist nun zwar nur beschränkt angängig. R. GrahmanN hat indessen für die letzte Vereisung unter Berücksichtigung dessen, daß das norddeutsche Inlandeis im Randgebiet sich aufwärts bewegte, ein Oberflächengefälle des Eises von 5\%oo zu Grunde gelegt (R. Grahmann 1937, S. 58), ein Betrag der zutreffend sein kann. Auch hier müßte ein gewisser Abschlag erfolgen, um dem gewundenen Weg des Schmelzwassers durch Spalten und Tunnel Rechnung zu tragen. Es kann also für die folgende hydrodynamische Betrachtung ein Gefälle des Wassers von 4,5\%

Die aufgeworfene Frage nach der Geschwindigkeit des subglaziären Wasserstroms entzieht sich aber auch dann, wenn das Gefälle bekannt ist, der exakten hydrodynamischen Berechnung, da die hydraulischen Berechnungsformeln Erfahrungswerte und Koeffizienten enthalten, die für die subglaziären Wasserwege fehlen und nicht beschafft werden können, sodaß auf zahlenmäßige Berechnung verzichtet werden muß.

Grundgedanke der Untersuchung: Es wird deswegen in der folgenden Untersuchung von den bekannten Werten offener Wasserläufe ausgegangen. Wenn sich hierbei ergeben wird, daßselbstunterdengünstigeren Widerstandsverhältnissen offener Wasserläufe einefürdie Schuttförderung ausreichende Wassergeschwindigkeit bei dem gegebenen Gefälle nicht entsteht, dann mußsie, da die Widerstände im subglaziären Wasserstrom ungleich größere sind, hier als a usgeschlossen betrachtet werden. Die Verhältnisse offener Wasserläufe werden also lediglich als Grenzwert verwendet. 
In Flüssen, also bei abwärts gerichtetem Gefälle, fängt die Geschiebeförderung, zunächst des Sandes, bei etwa $0,6-0,7 \mathrm{~m} / \mathrm{sek}$. Wassergeschwindigkeit an (van Rinsum 1950, S. 55). Für kiesigen Boden wird die Geschwindigkeit mit $1,0 \mathrm{~m} / \mathrm{sek}$. und für grobsteinigen Boden mit $1,25 \mathrm{~m} / \mathrm{sek}$. angegeben („Hütte“ I S. 479). Für eine Fließrichtung aufwärts, entgegen einem Gefälle, das beim Aufstieg aus der Ostseemulde recht beträchtlich ist, würde die Annahme einer erforderlichen Geschwindigkeit von doppelter Größe gerechtfertigt sein, es soll indessen zur Vorsicht nur eine Geschwindigkeit von $2 \mathrm{~m} / \mathrm{sek}$. zu Grunde gelegt werden. Das oben veranschlagte Gefälle von $4,5 \%$ entspricht etwa dem von Wasserläufen in Gebieten mit etwas stärkerer Oberflächenneigung, also zwischen Flachland und Gebirge (van Rinsum 1950a, S. 73). Untersuchungen an der Wertach, einem bei Augsburg in den Lech mündenden Nebenfluß, haben bei Hochwasser und bei einem Gefälle von 4\% eine mittlere Geschwindigkeit von $1,83 \mathrm{~m} / \mathrm{sek}$. ergeben und an der Sohle infolge der Reibung nur $0,57 \mathrm{~m} / \mathrm{sek}$. (van Rinsum 1950, S. 53).

Aus dem vorstehenden, von dem Verfasser van Rinsum beliebig gewählten Beispiel eines offenen Flußlaufes, der bei $4 \%$ oo Gefälle nur eine Sohlengeschwindigkeit von $0,57 \mathrm{~m} / \mathrm{sek}$. zeigt, geht hervor, daß in dem subglaziären Entwässerungssystem mit etwa 4,5\% Gefälle die Sohlengeschwindigkeit des Wassers noch unter $1 \mathrm{~m} / \mathrm{sek}$. bleiben mußte und zwar sogar dann, wenn die günstigeren Verhältnisse offener Flußläufe vorliegen würden. Die Mindestgeschwindigkeit des Wassers, bei der Förderung von Sand und Geröll eintritt, war oben mit $2 \mathrm{~m} / \mathrm{sek}$. festgestellt worden. Nun sind aber die Fließwiderstände im subglaziären Tunnel selbstverständlich mehrfach höher als in offenen Wasserläufen:

a) Zunächst ist die Reibung abhängig vom benetzten Umfang des Querschnittes des Wasserstroms. Im offenen Wasserlauf sind nur Sohle und Wände des Flußbettes benetzt, während im völlig gefüllten subglaziären Tunnel keine freie Oberfläche, sondern allseitig benetzter Umfang des Wasserstroms gegeben ist. Daraus resultiert schon fast eine Verdoppelung des äußeren Reibungswiderstandes.

b) $\mathrm{Zu}$ beachten ist ferner, daß der Reibungswiderstand etwa mit dem Quadrat der Wassergeschwindigkeit wächst (L. Prandtl 1949, S. 103). Bei einer Steigerung der Geschwindigkeit auf das erforderliche Maß würde also der Reibungswiderstand auf einen mehrfachen Betrag anwachsen.

c) Die subglaziären Tunnel waren vielfach verzweigt infolge der über den Untergrund verteilten Entstehung des Schmelzwassers durch den Schmelzvorgang und durch unterteilten Zufluß auf dem Spaltenwege. Die durch Richtungsänderungen entstehenden Potentialverluste waren also erheblich größer als beim offenen Flußlauf. Von noch größerer Bedeutung sind Verengungen und namentlich Erweiterungen der Tunnelquerschnitte. Je plötzlicher die Übergänge sind, um so größer wird der Potentialverlust. Am bedeutsamsten sind die Erweiterungen, da hier Neigung der Strömung zur Ablösung von der Wand besteht (L. Prandtl, S. 162 und „Hütte“, S. 482). Sie werden in den Tunneln in immer erneuter Wiederholung und mit immer weiterer Druckminderung auftreten, während sie in offenen Wasserläufen weniger bedeutsam sind, da hier die freie Oberfläche das Ausweichen gestattet.

Ergebnis: Aus der hydrodynamischen Betrachtung ergibt sich, daß die zur Förderung von Gletscherschutt durch die Tunnel aus der Ostseemulde aufwärts zu den Sandern erforderliche Wassergeschwindigkeit aus dem zur Verfügung stehenden Gefälle nicht erreicht werden konnte. Wenn schon im of fenen FluBlauf mit einem Gefälle von 4\% eine Sohlengeschwindigkeit 
von nur $0,57 \mathrm{~m} / \mathrm{sek}$. festgestellt wurde, so muß un te r g l e i c h e n V e r hä $1 \mathrm{t}$ $\mathrm{n}$ isse n die Geschwindigkeit bei $4,5 \%$ Gefälle offensichtlich weit unter $1 \mathrm{~m} / \mathrm{sek}$. bleiben und kann damit die zur Geröllförderung erforderliche Mindestgeschwindigkeit von $2 \mathrm{~m} / \mathrm{sek}$. noch nicht zur Hälfte erreichen. Die im vorstehenden aufgeführten Momente verursachen für den im Tunnel eingeschlossenen. Wasserstrom ohne freie Oberfäche eine Vervielfachung des Gesamtwiderstandes und damit eine Reduktion der Geschwindigkeit auf einen Bruchteil der erforderlichen. Die Gesamtdifferenz zwischen Soll und Ist bleibt so groß, daß sie auch durch eine im Rahmen des Denkbaren bleibende noch günstigere Gestaltung der geschätzten Zahlenwerte und der verwendeten Erfahrungswerte nicht behoben werden könnte. Nach der unter b) angeführten Erfahrungsregel würde bei Steigerung der Geschwindigkeit von $0,57 \mathrm{~m} / \mathrm{sek}$. auf $2 \mathrm{~m} / \mathrm{sek}$. der Reibungswiderstand auf den über 12-fachen Betrag ansteigen. Zu seiner Utberwindung müßte das Gefälle im gleichen Maß von 4 auf $49 \%$ gesteigert werden. Wenn man daneben noch die große Steilheit der meisten Tunneltäler in ihrem letzten Teil berücksichtigt, so wird in aller Deutlichkeit ersichtlich, da $\beta$ vom hydrodynamischen Gesichtspunkt aus die Tunnelhypothese aufgegeben werden muß.

Als Widerspruch zu diesem Ergebnis könnte die Beobachtung von „springbrunnenartigen" Erscheinungen an arktischen Gletschern angesehen werden. Aber einmal sind diese Beobachtungen nur seltener; zum anderen sind die Erscheinungen meistens recht unbedeutend. Am eindrucksvollsten ist der am Malaspina-Gletscher von Russel festgestellte 12-15 Fuß hoch aufspringende Wasserstrom. Es ist der Ausfluß eines $n u r$ a b wärts flie $\beta$ enden Stroms. Wenn ein Fluß gleicher Energie im Randgebiet, etwa im Bereich der westlichen Ostsee eingetroffen wäre, so würde seine Kraft doch bei weitem nicht ausreichen, um zu den Sandern in der Höhe des 10-20-fachen seiner Steighöhe hinauf zu gelangen. Der Malaspina-,,Springbrunnen“ spricht also bei seiner relativ geringen Steighöhe nicht für die Hypothese, sondern gegen sie.

Schließlich muß nochmals daran erinnert werden, daß der hydrodynamischen Untersuchung drei Voraussetzungen beigegeben waren (siehe III Abs. 1), die nur gemacht wurden, um bei der Untersuchung die der bisherigen Theorie günstigsten Verhältnisse unter Ausschaltung strittiger Fragen zu Grunde zu legen. Wenn man aber ein Tunnelsystem an der Sohle des Inlandeises annimmt, wie es auch die Hypothese selbst tut, dann kann aus den schon unter I 6 angegebenen Gründen wohl kaum die Annahme einer zum Kattegatt durchgehenden Verbindung des unter dem Eis befindlichen Entwässerungssystems als unbegründet abgelehnt werden. - Hinsichtlich der Spalten, die offenbar im Abschmelzgürtel sehr zahlreich gewesen sein werden (R. Grahmann 1937, S. 60f.), muß nochmals (siehe unter II) auf die Tatsache hingewiesen werden, daß nach dem Gesetz der kommunizierenden Röhren für die Größe des hydrostatischen Drucks nur die letzten randnahen Spalten in Betracht gezogen werden dürfen, wodurch die Erreichung genügenden Druckes von vornherein ausgeschlossen ist. Selbst wenn nur allein diese letztgenannte Voraussetzung fallen gelassen wird, was aus physikalischen Gründen unabweisbar ist, dann ist das hydrodynamische Untersuchungs-Ergebnis für die Hypothese noch ungünstiger: $\mathrm{Es}$ erscheint dann nicht nur die Förderung von Gletscherschutt, sondern sogar die Bewegung nur eines Wasserstromszur Sanderhöhe hinauf ausgeschlossen. Als Ergebnis der Untersuchung darf also festgestellt werden, daß die bisher gewöhnlich vertretene Theorie des in Tunnel- 
tälern aufsteigenden und Gletscherschutt mitführenden subglaziären Schmelzwassers nicht haltbar ist.

Die hydrodynamische Untersuchung behandelt einen Teil des gesamten Fragenkomplexes, der nur neben den auf morphologischem Gebiet (siehe I) gemachten Darlegungen zu betrachten ist. Man mag gegen die eine oder andere davon Bedenken erheben, aber das dargestellte Gesamtbild zeigt zum mindesten, daß das Problem einer erneuten Prüfung von geänderten Gesichtspunkten aus bedarf. Wenn die bisherige Hypothese aufgegeben wird, so ergibt sich die Notwendigkeit, die Erscheinungen des Eisrandes und der Sanderbildung anders als bisher zu erklären. Die Schuttförderung von unten zum Eisrand hinauf hat nicht auch fluviatil, sondern nur glaziär stattgefunden. Die Zerteilung der vor dem Rande aufgehäuften Schuttmassen sowie die Aufschüttung der Sander konnte nicht durch subglaziäre, sondern nur durch supraglaziäre oder inglaziäre Schmelzwasser erfolgen.

Den inglaziären Schmelzwasserläufen nach Art der Skizze P. Woldstedt's (1929, Abb. 40) wird eine erhöhte Bedeutung zugemessen werden müssen, aber nicht als Schuttförderer, sondern als Verteiler des vom Eis aufwärts bis zum Eisrand geschobenen Sandes und Gerölls. Eine gewisse Bestätigung bringen die Beobachtungen von E. Todtmann am Vatna-Jökull auf Island (E. Todtmann 1951, S. 335). Beim Rückzug des Eises bildeten sich zunächst Stauseen, die bei Erreichung genügender Wasserspiegelhöhe nach außen hin über die Sanderflächen entwässerten, aber ohne Schuttführung, da die Stauseen als Sandfang wirkten. In dem noch späteren Stadium fand eine subglaziäre Entwässerung zu den Belten hin statt (P. Woldsted 1950, S. 40 und 365), die indessen auch dann nur vorstellbar sein dürfte, wenn schon vorher ein subglaziäres Tunnelsystem bestanden hat, sodaß nur die letzte Verbindung von den Stauseen zu diesem hergestellt $\mathrm{zu}$ werden brauchte.

Mit den letzten Ausführungen ist das Ziel dieser Arbeit schon überschritten; denn es ist mit ihr nur beabsichtigt, die vielen Bedenken, die der Hypothese der Tunneltäler entgegenstehen, zusammenzufassen und durch hydrodynamische Erwägungen zu ergänzen. Die Lösung der vielen neu auftretenden Probleme, wenn man ohne die Hypothese auszukommen sucht, kann nur durch vereinigte Arbeit vieler in längerer Frist gelingen.

\section{Schriftenverzeichnis}

B. Brockamp: Die barometrische Höhenberechnung auf der deutschen Grönland-Expedition. - N. Jahrb. f. Geol. u. Pal. 1951, S. 53.

R. Grahmann: Form und Entwässerung des nordeurop. Inlandeises. - Mitt. d. Ges. f. Erdk. Leipzig 1937.

„Hütte“: Das Ingenieurs Taschenbuch. Berlin. 27. Auflage.

L. Prandtl: Führer durch die Strömungslehre. Braunschweig 1949.

vaN Rinsum: Der Abfluß in offenen natürlichen Wasserläufen. Berlin 1950. - Niederschlag und Abfluß im Regnitzgebiet. Beiträge zur Gewässerkunde. München 1950 [1950a].

E. M. Todtmann: Im Gletscherrückzugsgebiet des Vatna-Jökull auf Island 1950. Neues Jahrb. f. Geol. 1951, S. 335.

E. Werth: Fjorde, Fjärde und Föhrden. - Zeitschr. f. Gletscherk. 1908/9.

P. Woldstedt: Norddeutschland und angrenzende Gebiete im Eiszeitalter. Stuttgart 1950. - Das Eiszeitalter. 1929.

Ms. eingeg. 14. 3. 1952

Anschrift des Verf.: Reg.-Dir. a. D. Paul Jaspersen, Kiel, Goethestraße 8. 